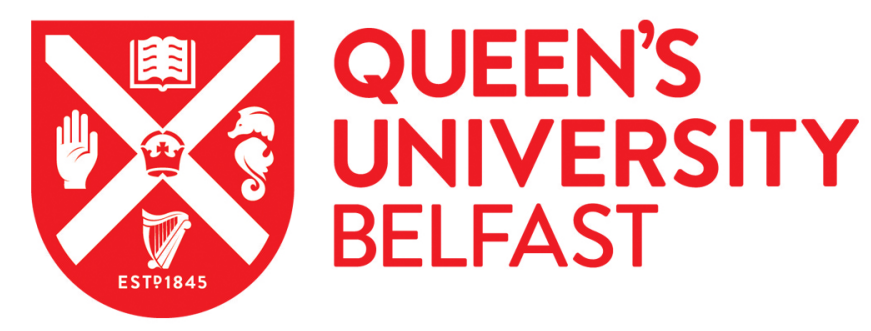

\title{
Energetic $\alpha$-particle sources produced through proton-boron reactions by high-energy high-intensity laser beams
}

Bonvalet, J., Nicolaï, P., Raffestin, D., D'Humieres, E., Batani, D., Tikhonchuk, V., Kantarelou, V., Giuffrida, L., Tosca, M., Korn, G., Picciotto, A., Morace, A., Abe, Y., Arikawa, Y., Fujioka, S., Fukuda, Y., Kuramitsu, Y., Habara, H., \& Margarone, D. (2021). Energetic a -particle sources produced through proton-boron reactions by high-energy high-intensity laser beams. Physical Review E, 103(5), [053202].

https://doi.org/10.1103/PhysRevE.103.053202

Published in:

Physical Review E

Document Version:

Publisher's PDF, also known as Version of record

Queen's University Belfast - Research Portal:

Link to publication record in Queen's University Belfast Research Portal

\section{Publisher rights}

(C)2021 American Physical Society

This work is made available online in accordance with the publisher's policies. Please refer to any applicable terms of use of the publisher.

\section{General rights}

Copyright for the publications made accessible via the Queen's University Belfast Research Portal is retained by the author(s) and / or other copyright owners and it is a condition of accessing these publications that users recognise and abide by the legal requirements associated with these rights.

Take down policy

The Research Portal is Queen's institutional repository that provides access to Queen's research output. Every effort has been made to ensure that content in the Research Portal does not infringe any person's rights, or applicable UK laws. If you discover content in the Research Portal that you believe breaches copyright or violates any law, please contact openaccess@qub.ac.uk. 


\title{
Energetic $\alpha$-particle sources produced through proton-boron reactions by high-energy high-intensity laser beams
}

\author{
J. Bonvalet $\odot,{ }^{1} \mathrm{Ph}$. Nicolaï $\odot,{ }^{1}$ D. Raffestin, ${ }^{1}$ E. D’humieres $\odot,{ }^{1}$ D. Batani, ${ }^{1}$ V. Tikhonchuk $\odot,{ }^{1,2}$ V. Kantarelou $\odot,{ }^{2}$ \\ L. Giuffrida $\odot,{ }^{2}$ M. Tosca $\odot,{ }^{2}$ G. Korn, ${ }^{2}$ A. Picciotto, ${ }^{3}$ A. Morace $\odot,{ }^{4}$ Y. Abe,${ }^{4}$ Y. Arikawa $\odot,{ }^{4}$ S. Fujioka $\odot,{ }^{4}$ Y. Fukuda, ${ }^{5}$ \\ Y. Kuramitsu, ${ }^{6}$ H. Habara $\odot,{ }^{6}$ and D. Margarone ${ }^{2,7}$ \\ ${ }^{1}$ Université de Bordeaux, CNRS, CEA, CELIA (Centre Lasers Intenses et Applications), UMR 5107, F-33405 Talence, France \\ ${ }^{2}$ ELI-Beamlines Center, Institute of Physics, Czech Academy of Sciences Za Radnici 835, 25241 Dolní Břežany, Czech Republic \\ ${ }^{3}$ Fondazione Bruno Kessler (FBK), Sensors and Devices - Micro Nano Facility, 38122 Trento, Italy \\ ${ }^{4}$ Institute of Laser Engineering, Osaka University, 2-6 Yamada-oka, Suita, Osaka, 565-0871, Japan \\ ${ }^{5}$ Kansai Photon Science Institute (KPSI), National Institutes for Quantum and Radiological Science and Technology (QST), \\ 8-1-7 Umemidai, Kizugawa-shi, Kyoto 619- 0215, Japan \\ ${ }^{6}$ Graduate School of Engineering, Osaka University, 2-1 Yamada-oka, Suita, Osaka, 565-0871, Japan \\ ${ }^{7}$ Centre for Plasma Physics, Queen's University Belfast, BT71NN Belfast, Northen Ireland, United Kingdom
}

(Received 20 November 2020; accepted 8 April 2021; published 5 May 2021)

\begin{abstract}
In an experiment performed with a high-intensity and high-energy laser system, $\alpha$-particle production in proton-boron reaction by using a laser-driven proton beam was measured. $\alpha$ particles were observed from the front and also from the rear side, even after a 2-mm-thick boron target. The data obtained in this experiment have been analyzed using a sequence of numerical simulations. The simulations clarify the mechanisms of $\alpha$-particle production and transport through the boron targets. $\alpha$-particle energies observed in the experiment and in the simulation reach 10-20 MeV through energy transfer from 20-30 MeV energy incident protons. Despite the lower cross sections for protons with energy above the sub-MeV resonances in the proton-boron reactions, $10^{8}-10^{9} \alpha$ particles per steradian have been detected.
\end{abstract}

DOI: 10.1103/PhysRevE.103.053202

\section{INTRODUCTION}

The proton-boron (p-B) fusion reaction has been investigated since the 1930s [1] and has received growing interest in recent years, due to multiple applications in different fields [2-7]. In the context of fusion for energy, this reaction has the advantage of releasing its energy with a very low neutron emission, which is attractive for the safety of a fusion reactor. However, this aneutronic fuel ignites at very high temperature, almost one order of magnitude higher than for the deuteriumtritium fuel [8]. To achieve significant fusion gain, it has been proposed to take advantage of secondary nuclear reactions from products of primary reactions, using natural boron or nitride-boron targets [9]. Although these cycles of reactions improve the energy balance, a fusion power reactor seems currently out of reach.

Among reaction products of $\mathrm{p}-\mathrm{B}$ fusion, $\alpha$ particles appear in large numbers and could be a valuable secondary source for different applications. For instance, theoretically $[10,11]$ and experimentally [12], the possibility to enhance proton-therapy effectiveness thanks to proton-boron reactions has been demonstrated. The method consists in injecting a solution containing boron into a tumor before the proton irradiation [13]. This process is already clinically used in neutron therapy [14-16]. $\alpha$ particles produced at low energies, having a short mean free path comparable with the cell size, significantly increase killing capacity. In addition, gamma emission produced in fusion reactions may be useful for online monitoring and evaluation of dose deposition $[17,18]$.

A promising approach to obtain bright $\alpha$-particle sources, has been proposed by Belyaev et al. [2] and uses laseraccelerated proton beams to initiate fusion reactions. An $\alpha$-particle yield of about $10^{5} \alpha /(\mathrm{sr}$ shot) has been measured in their experiment and this yield has been continuously enhanced, through various experiments $[4,5,19]$ up to a few $10^{10} \alpha /$ (sr shot) using a subnanosecond laser pulse with a modest intensity [6]. Main channels yielding $\alpha$ particles show resonances at low energies, 150 and $675 \mathrm{keV}$. In order to increase the number of $\alpha$ particles, the energies of laser-driven protons have to be in this range or protons need to shed their energy while slowing down in boron targets. Subsequent $\alpha$ particles likewise present an energy spectrum in the range of a few MeV. Due to their short ranges, their probability to escape from the target is small and they may constitute a bright secondary source only in the boron target itself.

Some medical or industrial applications require the use of alpha beams with energies of tens of MeV. Radioisotopes for imaging, therapy, or theranostics, such as scandium $\left({ }^{43} \mathrm{Sc}\right.$, ${ }^{44}{ }^{8} \mathrm{Sc},{ }^{44} \mathrm{Sc}$, and ${ }^{47} \mathrm{Sc}$ ) from $\alpha$-calcium or $\alpha$-potassium reactions, show maxima in cross sections for energies greater than $10 \mathrm{MeV}$ [20]. Classical medical cyclotrons do not reach these energies and only specific cyclotrons such as ARRONAX [21] can produce these radioisotopes. Laser-accelerated proton beams may produce energetic $\alpha$ particles through momentum 


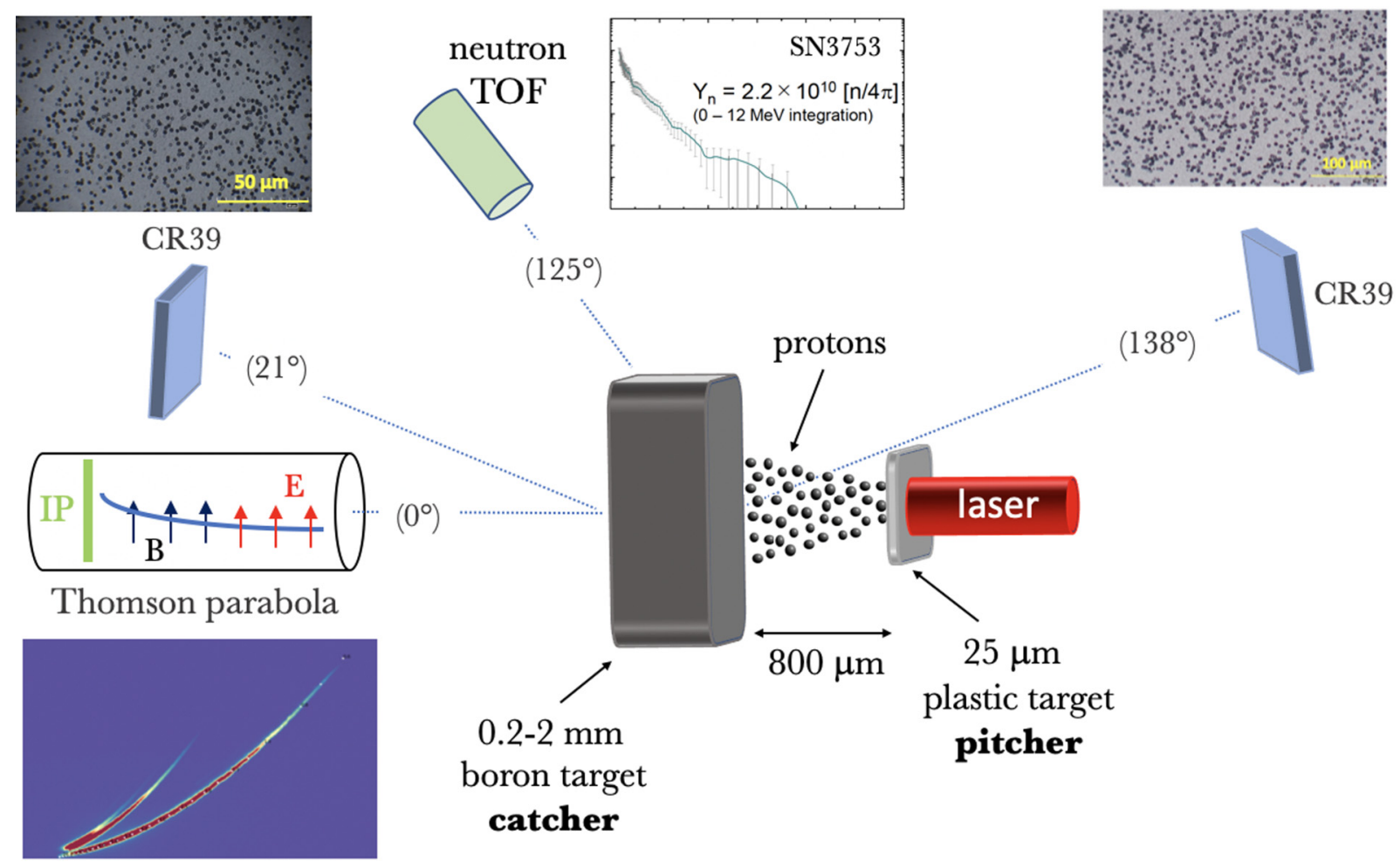

FIG. 1. Scheme of the experimental setup used to study the laser-driven proton-boron interaction at the high-energy and high-intensity LFEX laser. Protons are accelerated at the rear side of a thin plastic target and irradiate a boron-nitride or a boron target. $\alpha$-particle emission is measured with a Thomson parabola placed in the forward direction and CR-39 films placed at $21^{\circ}$ and $138^{\circ}$ with respect to the laser direction. A time-of-flight detector for neutron measurements was set at $125^{\circ}$.

transfer from kinetic energy of protons. In this work, we test numerically and experimentally the possibility to produce high-energy $\alpha$-particle beams at the front and also at the rear side of the boron target, in a pitcher-catcher scheme. We show that despite smaller cross sections compared to sub-MeV resonances, higher proton energies open new reaction channels and allow an additional energy transfer to $\alpha$ particles.

\section{EXPERIMENTAL SETUP AND MEASUREMENTS}

The experiment was carried out at the Institute of Laser Engineering at Osaka University, Japan using the short-pulse, high-intensity, high-energy PW class laser LFEX [22]. During the campaign, laser energies varied between 1.2 and $1.4 \mathrm{~kJ}$, in $2.7 \pm 0.45 \mathrm{ps}$ at the fundamental wavelength $(\lambda=1.05 \mu \mathrm{m})$. The focal spot size on target was $\sim 50 \mu \mathrm{m}$, full width at half maximum, corresponding to a laser intensity of $(2-3) \times 10^{19}$ $\mathrm{W} / \mathrm{cm}^{2}$. The laser illuminated a $(1 \mathrm{~mm} \times 1 \mathrm{~mm}) \times 25 \mu \mathrm{m}$ plastic target at normal incidence, in order to generate a high-energy proton beam through the process of target normal sheath acceleration [23]. The proton beam irradiated a $(5 \mathrm{~mm} \times 5 \mathrm{~mm}) \times 2 \mathrm{~mm}$ pure boron target or $(5 \mathrm{~mm} \times$ $5 \mathrm{~mm}) \times 0.2 \mathrm{~mm}$ nitride-boron targets placed at a distance of $800 \mu \mathrm{m}$ (pitcher-catcher scheme) and produced $\alpha$ particles through nuclear reactions as shown in Fig. 1. Different diagnostics were present for characterization of the primary proton source and secondary $\alpha$-particle stream at different angles, along with neutron production estimation.

\section{A. Thomson parabola}

A Thomson parabola (TP) was set behind the boron target, along the laser axis $\left(0^{\circ}\right)$ at a distance of $127 \mathrm{~cm}$. The Thomson parabola is based on the deflection of ions in magnetic and electric fields. Ions are collimated by a $300-\mu \mathrm{m}$ pinhole entrance, deflected according to their charge-to-mass ratio and their energies and were recorded on a Fujifilm BAS-TR imaging plate [24,25]. The whole geometry of the spectrometer was simulated numerically and calibrated, thus allowing one to characterize all ion spectra produced during the experimental campaign. Figure 2 presents particle tracks for the three main shots: plastic target alone, plastic target plus $200-\mu \mathrm{m}$ nitride-boron target, and plastic plus 2-mm pure boron target. On all images, proton tracks were clearly visible and up to $30 \mathrm{MeV}$ protons were observed to come out of the plastic target. After crossing the boron target, their energy and number decreased; however, 15-20 MeV protons were recorded even after the 2-mm-thick catcher. In the shot without a secondary boron target, carbon ions, $\mathrm{C}^{5+}$ and $\mathrm{C}^{6+}$, were observed with maximum energies up to $70 \mathrm{MeV}$. No $\alpha$ particles were detected on imaging plates whatever the boron target thickness. This means that $\alpha$-particle tracks are below the detection threshold. In Figs. 2(b) and 2(c), behind the tracks, a TP mechanical structure is visible by drop shadow showing the noise limit. For the 2-mm boron target, the proton track is barely visible, just above the threshold, likely indicating that $\alpha$ particles were not detectable. 


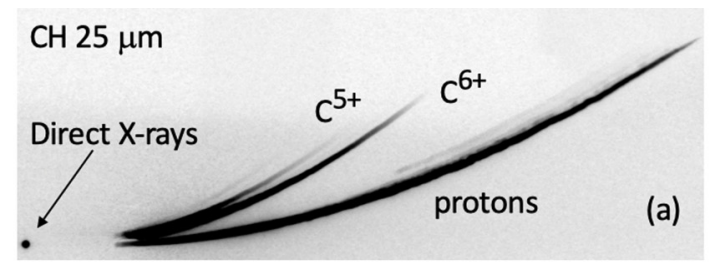

$\mathrm{CH} 25 \mu \mathrm{m}-200 \mu \mathrm{m} N-\mathrm{B}$

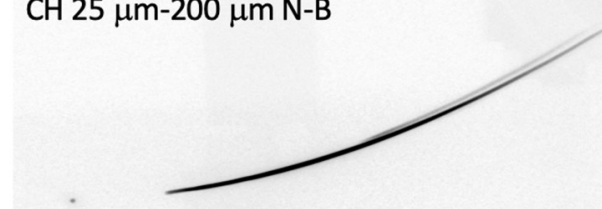

(b)
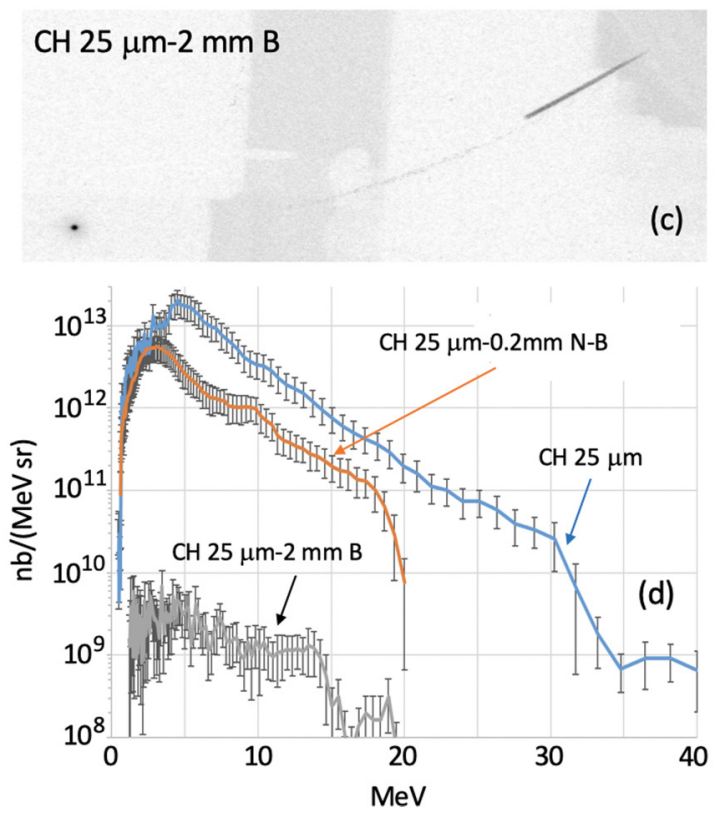

FIG. 2. Raw images obtained with Thomson parabola after 25$\mu \mathrm{m}$ C-H target (a), 25- $\mu \mathrm{m} \mathrm{C}-\mathrm{H}$ and $200-\mu \mathrm{m}$ N-B targets (b), and $25-\mu \mathrm{m} \mathrm{C}-\mathrm{H}$ and 2-mm B targets (c). Proton spectra inferred from raw images (d).

\section{B. CR-39 films}

CR-39 films were set at $21^{\circ}$ and $138^{\circ}$ with respect to the laser direction and at 35.9 and $48.3 \mathrm{~cm}$, respectively, from the target. Calibration of CR-39 was made at INFN [26] in Catania using a ${ }^{241} \mathrm{Am}$ thin source emitting $\alpha$ particles at $5.486 \mathrm{MeV}(85.2 \%)$ and $5.443 \mathrm{MeV}$ (12.8\%) [7]. By introducing appropriate filters in front of the source, the energy of particles was degraded to $2.6 \mathrm{MeV}$. In the experiment, CR-39 films were partially covered by $10-$ and $30-\mu \mathrm{m}$ Al layers to enhance the detected energy range up to $10 \mathrm{MeV}$ (Fig. 3) and allow building the $\alpha$-particle spectrum by energy ranges as shown in Fig. 4. Table I summarizes $\alpha$-particle measurements for different CR-39 film positions. For all targets, either from the front side or the rear side, a large number of energetic $\alpha$ particles has been detected. Above $10^{8} \alpha / \mathrm{sr}$ escaped from the front side $\left(138^{\circ}\right)$. From the rear side $\left(21^{\circ}\right)$, almost the same number of particles escaped after a 200- $\mu$ m target while the number is reduced by more than ten times after a $2-\mathrm{mm}$ boron target. The CR-39 films covered by $10 \mu \mathrm{m}$ of $\mathrm{Al}$ filter

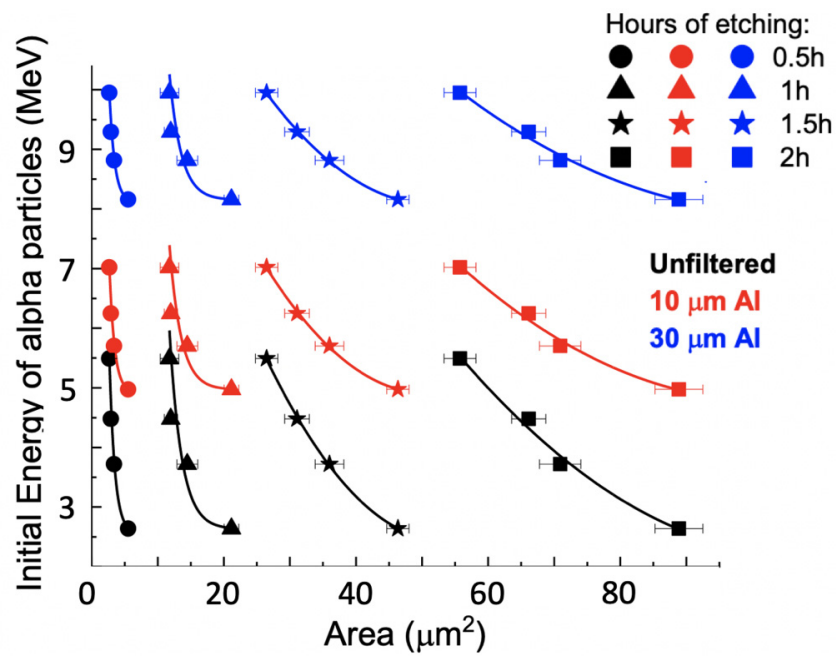

FIG. 3. Calibration curves that correlate the energy of the $\alpha$ particles before entering the Al filter (initial energy) with respect to the formatted area of the depicted tracks after $0.5,1,1.5$, and $2 \mathrm{~h}$ of etching.

(5-7 MeV range) set at $138^{\circ}$ for the N-B shot and at $21^{\circ}$ for the boron shot were unusable due to a technical issue which occurred during the installation process.

\section{Neutron time-of-flight detector}

Neutron spectroscopy was performed using a single channel neutron time-of-flight system consisting of a scintillatorbased neutron detector and a $2-\mathrm{GHz}$ digitizer. The detector was placed at a distance of $13.5 \mathrm{~m}$ at an angle of $125^{\circ}$ relative to the laser propagation axis. Typical spectra are presented in Fig. 5. Total neutron yields were between 2 and $7 \times 10^{10}$ neutrons in $4 \pi$ per shot. The presence of a secondary boron target did not significantly change either neutron yield or spectrum shape and maximum. This means the number of fusion neutrons produced in the boron target was negligible in comparison to the neutrons produced in reactions induced by protons and gammas emitted from the plastic target with the target chamber such as ${ }^{56} \mathrm{Fe}(\gamma, \mathrm{n})$ or ${ }^{56} \mathrm{Fe}(\mathrm{p}, \mathrm{n})$. It also implies that neutrons can hardly be used as a diagnostic of the $p-B$ fusion process in this experiment.

TABLE I. $\alpha$-particle counts/sr on CR-39.

\begin{tabular}{lcc}
\hline \hline CR position & \multicolumn{2}{c}{$138^{\circ} / 48.3 \mathrm{~cm}$} \\
\hline Energy range & $5.0-7.0 \mathrm{MeV}$ & $8.1-9.9 \mathrm{MeV}$ \\
B 2 mm & $1.3 \times 10^{8} \pm 4.5 \%$ & $4.44 \times 10^{7} \pm 50.0 \%$ \\
NB 0.2 mm & \multicolumn{2}{c}{$2.4 \times 10^{9} \pm 18.0 \%$} \\
CR position & \multicolumn{2}{c}{$21^{\circ} / 35.9 \mathrm{~cm}$} \\
\hline Energy range & $5.0-7.0 \mathrm{MeV}$ & $8.1-9.9 \mathrm{MeV}$ \\
B 2 mm & & $1.6 \times 10^{6} \pm 40.8 \%$ \\
NB 0.2 mm & $7.2 \times 10^{8} \pm 5.3 \%$ & $4.59 \times 10^{7} \pm 16.7 \%$ \\
\hline \hline
\end{tabular}



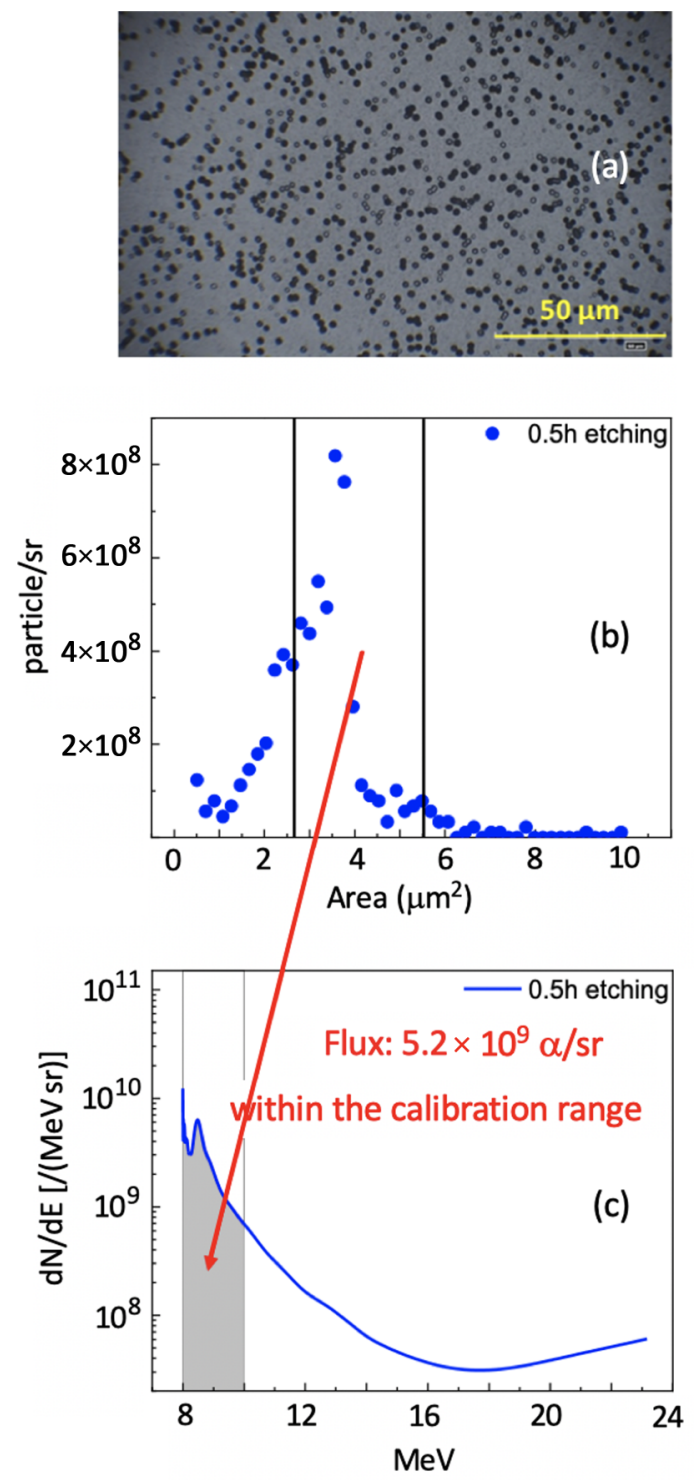

FIG. 4. (a) Microscopic image of $\alpha$-particle tracks after $0.5 \mathrm{~h}$ of etching. (b) Area distribution of the analyzed tracks; only the ones that are inside the vertical lines are within the calibration curves. (c) Energy distribution of the $\alpha$ particles; the total number of particles per steradian that correspond to the calibration range is $5.2 \times 10^{9} \alpha$ particles/sr.

\section{MODELING AND NUMERICAL TOOLS}

In order to simulate experimental results, a chain of numerical tools based on the particle-in-cell (PIC) code SMILEI [27] and the Monte Carlo (MC) code FLUKA [28] has been used. The first one allowed one to describe laser-plastic interaction and ion production at the rear side of the plastic pitcher. Then the proton characteristics defined in the phase space, giving position, energy, and direction of each particle, were used to initialize the MC code. The latter computes proton transport, energy losses, nuclear reactions, and secondary sources transport in a cold material of the secondary boron or nitride-boron catcher. This approach is appropriate for our experiment. The laser does not irradiate the boron target directly and the target remains cold.
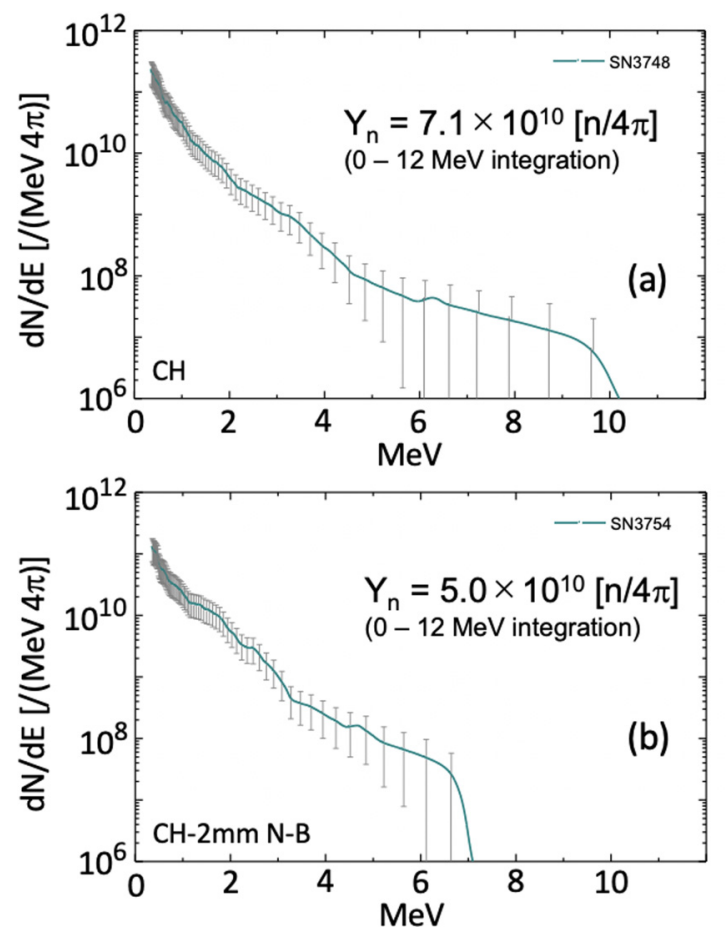

FIG. 5. Neutron spectra measured from plastic target alone (a) and plastic and 2-mm NB target (b).

\section{A. PIC simulations}

In PIC simulations, the LFEX laser parameters measured in the campaign were used: $1 \mu \mathrm{m}$ wavelength, a temporal Gaussian shape of $1.44 \mathrm{ps}$ duration at full width at half maximum (FWHM), and a focal spot on a target of $60 \mu \mathrm{m}$ at FWHM. Laser energy is $1.4 \mathrm{~kJ}$, yielding a laser intensity of $4.6 \times 10^{19}$ $\mathrm{W} / \mathrm{cm}^{2}$. The domain of simulation containing a $25-\mu \mathrm{m}$ plastic target is discretized in a $150 \times 180 \mu \mathrm{m}^{2}$ grid with a constant mesh size of $25 \times 25 \mathrm{~nm}^{2}$. Each cell with matter contains 50 particles. The ion density is fixed at $100 n_{c}$. Two-dimensional (2D) simulations last 8 ps and require $15 \mathrm{~h}$ using 9600 cores at the TGCC SKL computer center [29]. In the experiment, due to amplified spontaneous emission induced in the laser system, a preplasma in front of the target was present at the time of the main laser pulse interaction. This preplasma may modify the laser target interaction and consequently the spectrum of the protons escaping from the target rear side. Preplasmas with exponential density profiles with scale length varying from 2 to $20 \mu \mathrm{m}$ were tested and a characteristic length of $7.5 \mu \mathrm{m}$ provided a result in close agreement with the experiment. Figure 6(a) presents the comparison between the experimental spectrum measured by the Thomson parabola and the computed spectrum. Shapes, maximum energy, and proton number are in fair agreement. Simulation results may be used to access nonmeasured data. Indeed, the spectrum has been obtained along the laser axis and no information on angular distribution was accessible experimentally. Figure 6(b) shows the angular spectrum extracted from the simulation. It presents a maximum between $\pm 20^{\circ}$, which is typical for protons escaping from thin solid targets [30]. For the simulation of the second target with the MC code, the phase space of protons impacting on the boron target was built by combining 

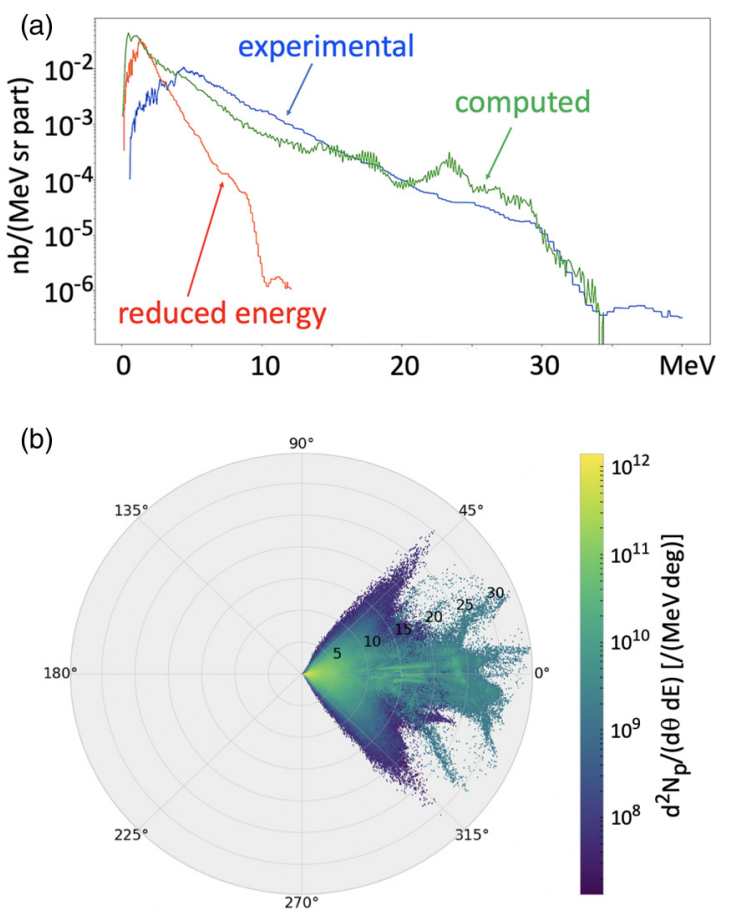

FIG. 6. Experimental (blue), computed (green), and reduced energy (red) proton spectra (a) and computed angular distribution in polar coordinates (b).

the measured energy spectrum with the computed angular distribution.

\section{B. Monte Carlo simulations}

\section{Proton energies and $\alpha$-particle sources}

The FLUKA code (version 2020.0.4) accurately models the interaction and transport of ions, electrons, photons, and neutrons from energies related to the physics of particles down to a few keV. FLUKA is continuously being benchmarked with models and experimental data concerning nuclear cross sections, including the proton-boron fusion reactions [31,32]. For low-energy protons, the most probable channel for $\alpha$-particle production is a sequential decay via ${ }^{8} \mathrm{Be}$ in its fundamental or excited state:

$$
\begin{aligned}
& p+{ }^{11} \mathrm{~B} \rightarrow \alpha_{0}+{ }^{8} \mathrm{Be} \rightarrow \alpha_{0}+\alpha_{01}+\alpha_{02}+8.59 \mathrm{MeV} \\
& p+{ }^{11} \mathrm{~B} \rightarrow \alpha_{1}+{ }^{8} \mathrm{Be}^{*} \rightarrow \alpha_{1}+\alpha_{11}+\alpha_{12}+5.65 \mathrm{MeV}
\end{aligned}
$$

The probability of the latter reaction is ten times more probable and presents resonances at low energies, the main one at $675 \mathrm{keV}$ (Ref. [33]). For higher proton energies the cross section slowly decreases but remains high enough to be a source of a large number of $\alpha$ particles. In addition, for higher energies, additional reactions become more probable and may be sources of $\alpha$ particles. All fusion reactions initiated in the FLUKA computations by the proton spectrum shown in Fig. 6(a) are listed in the Appendix. In the experiment, the pure boron target is composed of ${ }^{11} \mathrm{~B}$ at $80 \%$ and ${ }^{10} \mathrm{~B}$ at $20 \%$. Some of the numerous $\mathrm{p}^{-10} \mathrm{~B}$ reactions are sources of $\alpha$ particles (see Appendix). Likewise, proton-nitrogen interaction also produces $\alpha$ particles, although in a smaller number and possible secondary fusion cycles, as shown in [4]. The first preliminary run consisted in calculating the source of $\alpha$ particles from each component of a nitride-boron target composed by ${ }^{14} \mathrm{~N}(50 \%) /{ }^{11} \mathrm{~B}(40 \%) /{ }^{10} \mathrm{~B}(10 \%)$. From FLUKA computation, each component is a source of $\alpha$ particles and even if boron is the main source $\left(78.1 \%\right.$ from ${ }^{11} \mathrm{~B}$ and $12.8 \%$ from ${ }^{10} \mathrm{~B}$ ), the number of $\alpha$ particles produced in nitrogen reactions is not negligible (about $9 \%$ of the total). The number of produced neutrons and photons is significant, and even though proton-boron reaction is considered aneutronic, the relative neutron yield is $7.4 \%$ with respect to the number of $\alpha$ particles.

\section{Effects of a reduced energy proton spectrum}

Previous studies $[2,4-6,19]$ reported on the observation of $\alpha$ particles emitted from the front side, directly irradiated by the laser or by the proton beam. In the context of fusion for energy, these studies aimed at obtaining a high fusion reaction yield and not necessarily high $\alpha$-particle energies. Due to the presence of resonances at sub-MeV energies in the proton-boron cross section, high-energy proton fluxes were not mandatory, and even counterproductive since the cross section decreases at higher proton energies. Thereby, at modest laser intensity $\left(\sim 10^{16} \mathrm{~W} / \mathrm{cm}^{2}\right)$ Giuffrida et al. [6] observed about $10^{11} \alpha$ particles by directly illuminating a boron target. Closer to our experimental setup, Baccou and co-workers [4] used the pitcher-catcher scheme to induce the $\mathrm{p}-\mathrm{B}$ reactions in a secondary target. The proton spectra measured in their experiment had maximum energies between 3.5 and $6.5 \mathrm{MeV}$; much smaller than in our experiment. The goal of the present study, is to produce at the rear side of the boron target, an $\alpha$-particle beam by transferring proton kinetic energy to create an energetic and bright secondary source. Thus one has to verify that using high-energy protons required to get energetic $\alpha$ particles, their amount is not strongly reduced. A comparison, under the same conditions, with a reduced energy proton spectrum allows one to address this issue. The reduced spectrum, shown in Fig. 6, with a maximum of $10 \mathrm{MeV}$, has been injected into the $200-\mu \mathrm{m}$ N-B target. In this case, the contribution in $\alpha$-particles production of boron and specifically of ${ }^{11} \mathrm{~B}$ is more important: $91.4 \%$ from ${ }^{11} \mathrm{~B}, 6.2 \%$ from ${ }^{10} \mathrm{~B}$, and $2.2 \%$ from ${ }^{14} \mathrm{~N}$. Neutrons have a relative yield of only $2.3 \%$ with respect to the $\alpha$-particles number. These variations come from different effects. High proton energies increase the number of possible nuclear reactions or increase their probability. In particular, the reaction $p+{ }^{10} \mathrm{~B} \rightarrow \alpha+{ }^{7} \mathrm{Be}$ presents a maximum of hundreds of millibarns for proton energies between 5 and $12 \mathrm{MeV}$ and the reaction $p+{ }^{14} \mathrm{~N} \rightarrow \alpha+{ }^{11} \mathrm{C}$ for energies between 5 and $30 \mathrm{MeV}$. For the same reasons, neutron yields for lower energies are much smaller due to energy thresholds in cross sections.

The total number of $\alpha$ particles produced in the target is smaller with the reduced energy proton spectrum. This result looks counterintuitive because the $\mathrm{p}-{ }^{11} \mathrm{~B}$ cross section presents a maximum at $675 \mathrm{keV}$. However, sub-MeV protons have a very small probability to interact, due to their very short range in the boron target (around $7 \mu \mathrm{m}$ at $675 \mathrm{keV}$ ). In addition, the main resonance at $675 \mathrm{keV}$ is relatively narrow and protons have this energy only for an infinitesimal path length. 


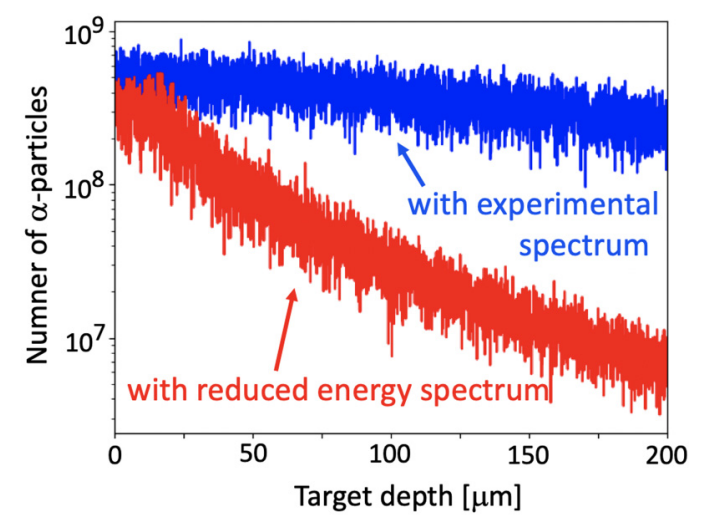

FIG. 7. Number of $\alpha$ particles as a function of the target depth produced with the experimental (blue) and reduced energy (red) proton spectra.

Higher-energy protons penetrate more deeply and even if the cross section is smaller, they integrate the interaction probability over a longer range and some of them finally also go through the sub-MeV resonance. Figure 7 shows where $\alpha$ particles are produced inside the $200-\mu \mathrm{m}$ N-B target for both proton spectra. Due to the proton mean free path, the number of $\alpha$ particles strongly decreases versus depth target for the reduced energy spectrum. With the experimental spectrum, the whole target thickness is a source of $\alpha$ particles. Thus, despite the presence of resonances at low energies, higherenergy protons produce a large amount of $\alpha$ particles and in addition allow kinetic energy transfer.

\section{Kinetic energy transfer from protons to $\alpha$ particles}

The number of escaping $\alpha$ particles from both sides of the boron target is an important criteria if one aims at generating a brilliant $\alpha$-particle source. Figure 8 presents spectra of $\alpha$ particles exiting from the rear and the front sides of the $\mathrm{N}-\mathrm{B}$ target. Two times less $\alpha$ particles can escape from the
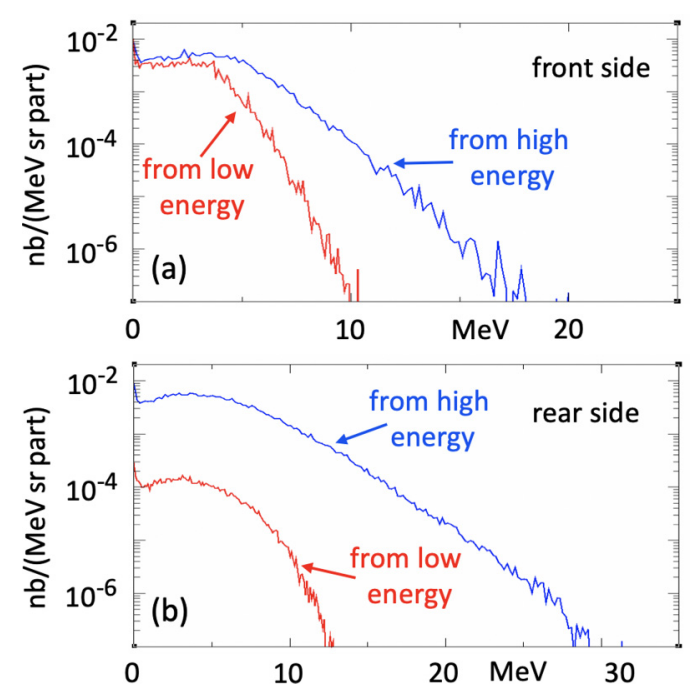

FIG. 8. $\alpha$-particle spectra escaping from the front (a) and the rear (b) sides. In red induced by low-energy and blue by high-energy spectra. front side when the reduced energy spectrum is injected. This ratio goes down to 50 times, at the rear side. Another important point concerns the energies of $\alpha$ particles. Thanks to a large kinetic energy transfer between protons and $\alpha$ particles, the maximum energy reaches $29 \mathrm{MeV}$ at the rear side and about $18 \mathrm{MeV}$ at the front one. Using the reduced spectrum, they only reach 13 and $10 \mathrm{MeV}$ at the rear and front sides, respectively.

The energy transfer can be estimated beforehand from energy and momentum conservation equations. By combining them, one obtains an equation for kinetic energy of primary $\alpha$ particle in the nuclear reactions (1) and (2):

$$
\begin{aligned}
\left(m_{\mathrm{Be}}+m_{\alpha}\right) E_{\alpha} & -2 \sqrt{m_{p} m_{\alpha} E_{p} E_{\alpha}} \cos (\theta) \\
& +\left(m_{p} m_{\mathrm{Be}}\right) E_{p}+m_{\mathrm{Be}}\left(E_{\mathrm{Be}}^{*}-Q\right)=0,
\end{aligned}
$$

where $m_{x}$ and $E_{x}$ are the mass and the kinetic energy of the particle $x, Q$ the energy released in the reaction, $E_{\mathrm{Be}}^{*}$ the excitation energy of $\mathrm{Be}$, and $\theta$ the $\alpha$-particle emission angle relative to the proton direction. Considering the most probable channel of proton-boron reaction, via the excited state of $\mathrm{Be}(Q=5.65 \mathrm{MeV})$, one obtains in the forward direction $(\theta=0)$ a maximum $\alpha$-particle energy of $\sim 32 \mathrm{MeV}$ with our experimental proton spectrum and $\sim 14 \mathrm{MeV}$ with the reduced spectrum. In the backward direction $(\theta=\pi)$, calculated $\alpha$ particle energies are $\sim 16 \mathrm{MeV}$ and $\sim 7.5 \mathrm{MeV}$, respectively. These values are in agreement with $\mathrm{MC}$ results and show a significant dependence of $\alpha$-particle energy with the proton energy. The range of $\alpha$ particles inside the catcher target strongly depends on their energies. We notice, for instance, that a $5-\mathrm{MeV} \alpha$ particle can only cross $20 \mu \mathrm{m}$ of boron target, whereas a $20-\mathrm{MeV}$ one can cross $180 \mu \mathrm{m}$.

The kinetic energy transfer also affects the $\alpha$-particle distribution. The reactions via the ${ }^{8} \mathrm{Be}$ states produce well-defined energies for primary $\alpha$ particles, and their peak shapes reflect the total widths of these states. Typical spectra have been measured and are presented, for instance, in Ref. [34]. The ${ }^{8} \mathrm{Be}$ breakout into two secondary $\alpha$ particles leads to a continuous energy distribution. Energies of $\alpha_{0}$ and $\alpha_{1}$ depend on the released nuclear reaction energy and on incident proton energy. A monokinetic energy proton beam like those produced by a cyclotron, leads to a structured spectrum, but in our case laser-driven protons present an exponential energy shape which smooths structures in a continuous energy spectrum.

\section{Proton spectra computations}

Experimental shot-to-shot fluctuations in laser parameters (energy and pulse duration) may affect the proton spectrum produced in the plastic target. Consequently, the spectrum of protons impacting the boron target, may vary from one shot to another. As mentioned above, the protons escaping from the boron targets have been measured along the laser axis with the TP. A good agreement between simulation and experimental results would give confidence in the injected proton spectrum at least for intermediate and high energies. Figure 9 shows proton spectra after crossing the $200-\mu \mathrm{m}$ N-B target and the 2-mm pure boron target, computed with the FLUKA code. The injected spectrum is in /(MeV sr) and is normalized to one incident particle. A comparison with the measured proton spectrum shown in Fig. 2 indicates a similar 


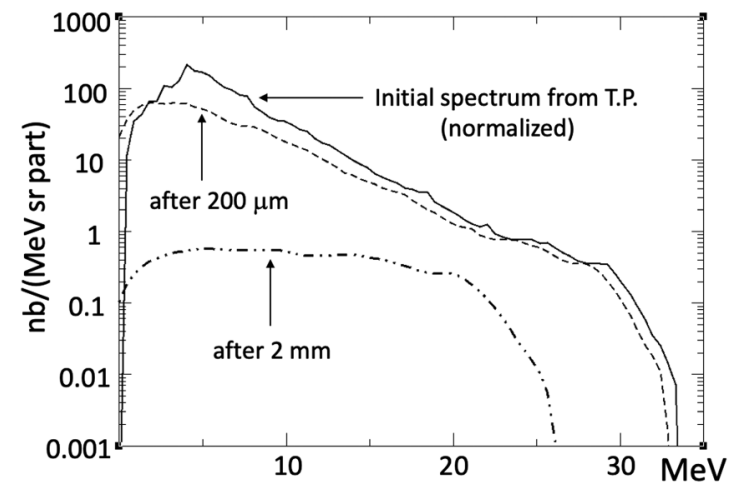

FIG. 9. Injected spectrum (solid) and computed ones after crossing the $200-\mu \mathrm{m} \mathrm{N}-\mathrm{B}$ target (dashed) and 2-mm boron target (dotted-dashed).

trend but the cutoff energy is not in agreement. After crossing a $200-\mu \mathrm{m}$ target in the simulation, the spectrum is mainly changed at low energy, while at high energies it is almost not modified. This means that in order to reproduce the experimental spectrum, for this target thickness, the spectrum of injected protons should have a cutoff energy close to $20 \mathrm{MeV}$. Noting that the stopping length of a $5-\mathrm{MeV}$ proton is $180 \mu \mathrm{m}$, only energies above this value can be validated. Figure 10 presents the incident proton spectra, which allowed producing the best agreement with experimental spectra measured after $200-\mu \mathrm{m}$ and $2-\mathrm{mm}$ targets. For the $200-\mu \mathrm{m}$ target, $18 \%$ of incident protons can cross and escape from the rear side. In the experiment, $2.6 \times 10^{13} / \mathrm{sr}$ protons have been measured after the target corresponding therefore to $1.4 \times 10^{14} / \mathrm{sr}$ injected protons. For the 2-mm boron target, an initial spectrum having a cutoff energy of $26 \mathrm{MeV}$ gives a fairly good agreement. After crossing target, only $0.2 \%$ of protons can escape. By contrast to the $200-\mu \mathrm{m}$ catcher target, a small change in the
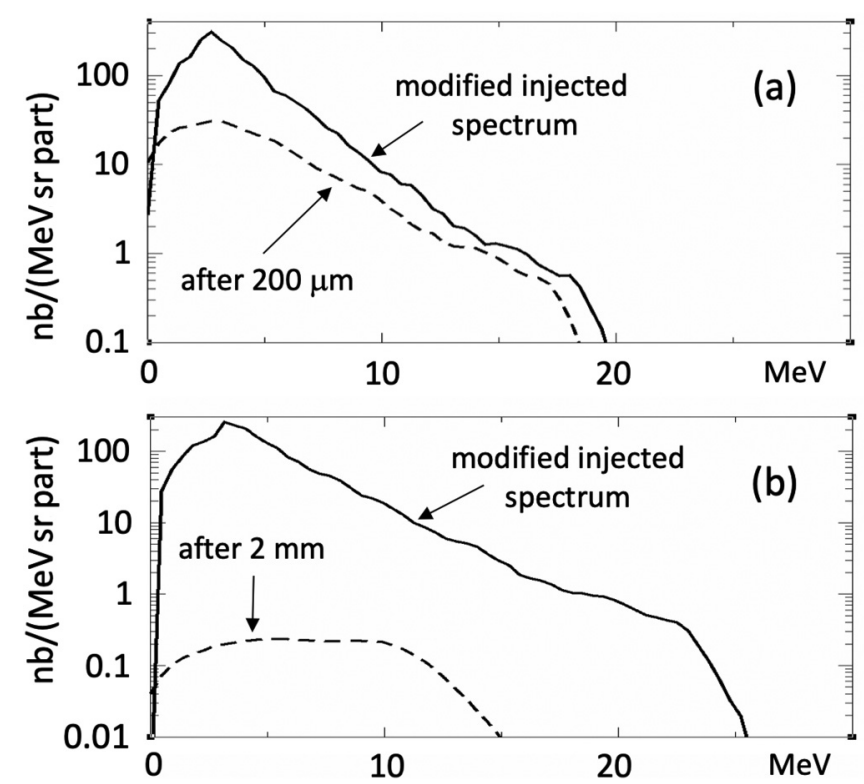

FIG. 10. Injected proton spectrum (solid) and escaped proton spectrum (dashed) for the $200-\mu \mathrm{m} \mathrm{N}-\mathrm{B}$ target (a) and the 2-mm boron target (b). incident cutoff energy can drastically modify the spectrum of protons after the $2-\mathrm{mm}$ catcher target. In the experiment, $3 \times 10^{10} /$ sr protons have been measured, corresponding to $1.5 \times 10^{13} / \mathrm{sr}$ incident protons. The adjustment of the incident proton spectrum for reproducing the experimental data implies a variation of the cutoff energy between 21 and 33 $\mathrm{MeV}$ and proton number by an order of magnitude from $1.5 \times 10^{13} /$ sr to $1.4 \times 10^{14} / \mathrm{sr}$. It is important to underline that only a part of the proton spectrum can be compared to the experimental one, especially for the thick target. It is then possible that the total proton number is modified in this low-energy range. Another important point concerns the track induced by protons after crossing a 2-mm boron target. As shown in Fig. 2, the signal is just above the detection threshold and hardly visible. Consequently, any particles reaching the TP with a lower flux do not produce any visible track and so cannot be measured.

\section{5. $\alpha$-particle spectra computations}

As the initial proton spectra is fixed for each target thickness, nuclear reactions and $\alpha$-particle production can be computed and compared to the experimental data for each shot separately for the target rear side. There is a larger uncertainty for the $\alpha$-particle production from the front side, because of a low-energy portion of proton spectra that was not measured. Figure 11 presents angular distributions and energy spectra of $\alpha$ particles for both targets. Particles escaping from the rear side are more energetic and reach $20 \mathrm{MeV}$ after a $200-\mu \mathrm{m}$ target, whereas from the front side, the cutoff energy is lower; about $12 \mathrm{MeV}$. After a 2-mm target, because protons lost a large part of their energy into the target, the kinetic energy transfer to $\alpha$ particles is smaller and the front and rear sides present similar maximum energy. From energy and momentum balance equations [Eq. (3)], maximum energies at the front and the rear sides may be confirmed. Considering the injected and escaped proton spectra (Fig. 10) for each target, the energy lost crossing the target is taken into account. One obtains for the 2-mm boron target, $\alpha$-particles energies of 17 and $14 \mathrm{MeV}$ at the front and the rear sides and for the 200- $\mu \mathrm{m}$ nitride-boron target, 11.5 and $18 \mathrm{MeV}$, respectively. These values are in good agreement with the computed ones. For the 200- $\mu \mathrm{m}$ target, for one incident proton, $7.3 \times 10^{-6} \alpha$ particles exit the target rear side and only $2.4 \times 10^{-7}$ from the rear side of the $2-\mathrm{mm}$ target, which is 30 times less. The low-energy part of the proton spectra used in MC simulations was not experimentally validated. A variation of the number of protons in the energy range below $5 \mathrm{MeV}$ can substantially modify the $\alpha$-particles production from the front side. With this caveat, our simulations show that, for one incident proton, $1.1 \times 10^{-5} \alpha$ particle exit from the front side of the N-B target and $2.6 \times 10^{-5}$ from the 2 -mm boron target. To go back to particle numbers, assuming $\sim 10^{14} / \mathrm{sr}$ incident protons, $\sim 7 \times 10^{8} / \mathrm{sr} \alpha$ particles exit from the $200-\mu \mathrm{m} \mathrm{N}-\mathrm{B}$ target rear side and $\sim 2.5 \times 10^{7} / \mathrm{sr}$ from the 2 -mm boron target.

\section{Discussion}

To compare these numbers with the experiment, the same energy range can be chosen. Table II summarizes results and can be compared with the experimental data given in Table I. 

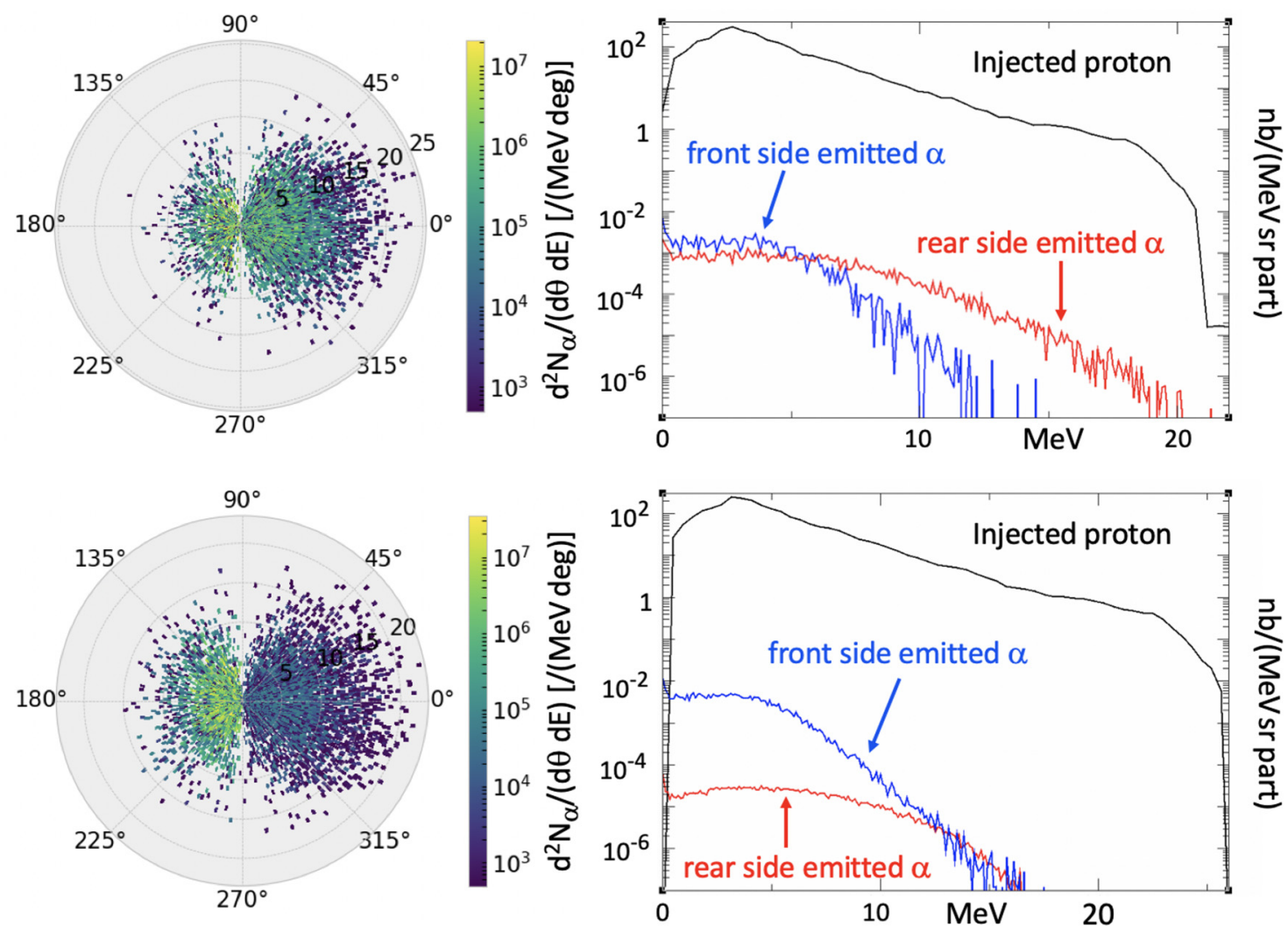

FIG. 11. $\alpha$-particle angular distributions (left) and energy spectra (right) for the 200- $\mu \mathrm{m} \mathrm{N}$-B target (top) and the 2-mm B target (bottom). Laser comes from the left side. $\alpha$-particle rear side emission is in red and front side emission in blue.

For the $200 \mu \mathrm{m}$ we predicted $1.5 \times 10^{8} / \mathrm{sr} \alpha$ particles in the 5-7 MeV range and $9.5 \times 10^{7} / \mathrm{sr}$ in the $8-10 \mathrm{MeV}$ range. For the 2-mm target, we calculate $4.9 \times 10^{6} / \mathrm{sr} \alpha$ particles in the range $8-10 \mathrm{MeV}$. Comparing these numbers with the data in Table I, the agreement for the observation angle of $21^{\circ}$ is rather good. Concerning the front side, for the $2-\mathrm{mm}$ target, the simulation gives in the $5-7 \mathrm{MeV}$ range $3.7 \times 10^{8} / \mathrm{sr} \alpha$ particles and $3.3 \times 10^{7} / \mathrm{sr}$ in the $8-10 \mathrm{MeV}$ range. These numbers are in agreement with the observation for the angle of $138^{\circ}$. For the $200-\mu \mathrm{m}$ target, in the simulation, $1.5 \times 10^{8} / \mathrm{sr} \alpha$ particles have been produced from the front side in the energy range of 5-7 MeV and $7.8 \times 10^{7} / \mathrm{sr}$ in the $8-10 \mathrm{MeV}$ range; a similar number was calculated for a 2 -mm boron target but it is smaller than the measured one. This difference could be explained by an underestimation of the number of incident

TABLE II. $\alpha$-particle counts/sr in simulations.

\begin{tabular}{lcc}
\hline \hline CR position & \multicolumn{2}{c}{ Front side } \\
\hline Energy range & $5.0-7.0 \mathrm{MeV}$ & $8.0-10.0 \mathrm{MeV}$ \\
B $2 \mathrm{~mm}$ & $3.7 \times 10^{8}$ & $3.3 \times 10^{7}$ \\
NB $0.2 \mathrm{~mm}$ & $1.5 \times 10^{8}$ & $7.8 \times 10^{6}$ \\
CR position & \multicolumn{2}{c}{ Rear side } \\
\hline Energy range & $5.0-7.0 \mathrm{MeV}$ & $8.0-10.0 \mathrm{MeV}$ \\
B 2 mm & $5.4 \times 10^{6}$ & $4.9 \times 10^{6}$ \\
NB $0.2 \mathrm{~mm}$ & $1.5 \times 10^{8}$ & $9.5 \times 10^{7}$ \\
\hline \hline
\end{tabular}

protons in the low-energy part of the spectrum which was not measured in the experiment.

Considering the relatively high proton energy, there are many nuclear sources of $\alpha$ particles in our experiment and some of them may take place in a plastic target through the proton-carbon reactions. The CR-39 films were placed in the forward and backward directions and measurements account for all $\alpha$-particle sources. However, in our experimental geometry based on a pitcher-catcher configuration, $\alpha$ particles emitted at the rear side of the plastic target have to cross the boron target to reach the CR-39 film set behind it, at the angle of $21^{\circ}$. Even for the thinnest boron target $(200 \mu \mathrm{m}), \alpha$ particles need to have at least $21 \mathrm{MeV}$ to cross it. For the CR-39 film set at $138^{\circ}$ (front side), no obstacle can prevent $\alpha$ particles produced at the front side of the plastic target to reach the CR39 film. The proton-carbon nuclear reaction producesthree $\alpha$ particles, $\mathrm{p}+{ }^{12} \mathrm{C} \rightarrow 3 \alpha+\mathrm{p}^{\prime}$ with possible transient steps through ${ }^{8} \mathrm{Be}$ or ${ }^{9} \mathrm{~B}$ (ground or excited states). This nuclear reaction is endothermal like the proton-nitrogen reaction and the energy threshold is more than $7 \mathrm{MeV}$, meaning that only high-energy protons can trigger this reaction. As measured in the experiment, protons escaping from the plastic target have an energy range up to $30 \mathrm{MeV}$. So proton-nitrogen reactions in the NB target, with $Q \sim-2.9 \mathrm{MeV}$, can be easily triggered. The $\mathrm{p}-\mathrm{C}$ reactions have to be triggered inside the plastic target and the proton acceleration has to take place at the front side of the target. The so-called hole boring process may accelerate protons at the front side of the target [35]. However, in the intensity range reached at LFEX and with a solid 


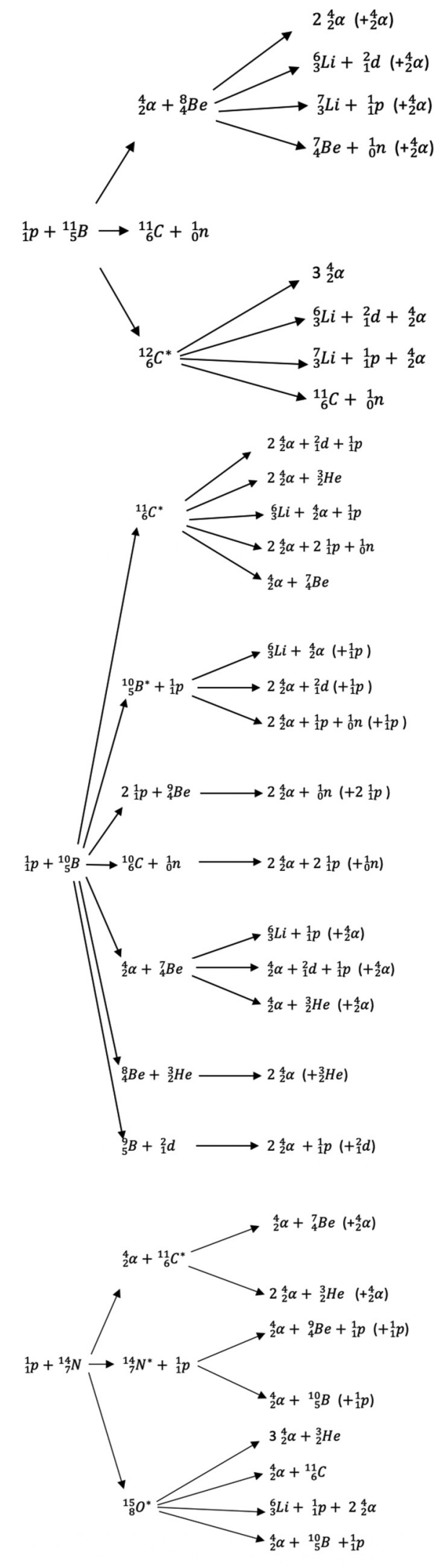

FIG. 12. Reactions induced in FLUKA computation by experimental proton spectrum. target, the proton energy remains weak. The maximum proton velocity can be estimated [35]: $v_{p}=2 c \sqrt{\frac{m_{e}}{m_{p}} \frac{Z}{A} \frac{n_{c}}{n_{e}}} a_{0}$, where $a_{0}$ is the laser field amplitude and $n_{c}$ the critical density. In our experimental conditions, with a solid target, the maximum energy is of the order of a few tens of keV. However, a laser prepulse partly ablates the plastic target front side, inducing a density gradient. Relativistic laser self-focusing up to $\gamma n_{c}$ and electron acceleration in such a preplasma result in a more efficient ion acceleration to a maximum energy of 7-8 MeV. The kinetic energy of the reaction products is on the order of $1 \mathrm{MeV}$; it is shared between the exiting proton and three $\alpha$ particles. Such low-energy $\alpha$ particles have to exit from the plastic target and cross the aluminum layer set in front of the CR-39 films. To cross $10 \mu \mathrm{m}$ of aluminum, an $\alpha$ particle needs to have a minimum energy of $2.9 \mathrm{MeV}$. In these conditions, $\alpha$ particles produced in the plastic target cannot contribute to the CR-39 films at the front side or at the rear side.

\section{CONCLUSION}

This work presents experimental results on $\alpha$-particle production in $\mathrm{p}-\mathrm{B}$ reactions by using a proton beam accelerated in the interaction of a high-intensity, high-energy laser with a thin plastic target. This bright and energetic proton source has been used to irradiate boron or nitride-boron targets of different thicknesses. The energy distribution and absolute number of protons and $\alpha$ particles has been measured in several directions from the front and rear sides of the targets. The observation of the $\alpha$-particle emission at the target rear side can be very important in view of the development of protonboron driven bright $\alpha$-particle sources. Indeed, the catcher target acts as a filter, removing ion species which are present on the target front side. The data obtained in this experiment have been analyzed using a sequence of numerical simulations with PIC and MC codes. The proton energy distributions used in $\mathrm{MC}$ simulations were adjusted for each shot by comparison with the measured proton spectra before and after the catcher target. The simulations clarify the mechanisms of $\alpha$-particles production and transport through the boron targets. $\alpha$ particles of high energies observed in the experiment are produced by energy transfer from incident protons. In contrast to other experiments, $\alpha$ particles are observed from the front side and also from the rear side, even after a 2-mm-thick boron target. By comparing with experiments at lower proton energies and despite the $\mathrm{p}-{ }^{11} \mathrm{~B}$ cross-section peak at low energy, still $10^{8}-$ $10^{9} \alpha$ particles per steradian have been detected. Due to the fact that the cross section of competitive nuclear reactions increases at higher energies, contributions of ${ }^{10} \mathrm{~B}$ and ${ }^{14} \mathrm{~N}$ in $\alpha$-particles production are no longer negligible. More importantly, energetic protons may produce more $\alpha$ particles, just considering $\mathrm{p}^{-11} \mathrm{~B}$ reaction. This reaction has high resonances at sub-MeV energy of protons. However, sub-MeV protons have a very small probability to interact, because they have a short range (a few microns) in the boron target. In addition, the main resonance at $675 \mathrm{keV}$ is narrow and protons have this energy only for an infinitesimal path length. Higher-energy protons travel more inside the boron target, and thus interact over a longer range. Furthermore, a part of these high-energy 
protons is finally slowed down to sub-MeV energies, thus reaching the proton-boron fusion main resonance.

In the present experiment, carried out in the pitcher-catcher geometry, the best results were obtained with a $200-\mu \mathrm{m}$ boron target, but in the future, this thickness could be better adapted to the proton spectrum to find a trade-off between a large number of nuclear reactions and limited energy lost by protons crossing the target.

\section{ACKNOWLEDGMENTS}

The PIC simulation work was granted access to HPC resources of TGCC under the allocation A0010506129 made by GENCI and under the allocation 2017174175 made by PRACE. This work has been carried out in the framework of regional project POPRA2 supported by the New Aquitaine Regional Council (Convention No. 18003358). This work has been carried out within the framework of the EUROfusion Enabling Research Project: ENR-IFE19.CEA-01 "Study of Direct Drive and Shock Ignition for IFE: Theory, Simulations, Experiments, Diagnostics Development" and has received funding from the Euratom research and training programme 2019-2020 under Grant Agreement No. 633053. The views and opinions expressed herein do not necessarily reflect those of the European Commission. This work was partially supported by JSPS KAKENHI No. 19H00668.

\section{APPENDIX}

Nuclear reactions involved in FLUKA computations depend on initial proton energies. The proton spectrum presented in Fig. 6(a) induces the reactions shown in Fig. 12.
[1] M. L. E. Oliphant and E. Rutherford, Proc. R. Soc. London, Ser. A 141, 259 (1933).

[2] V. S. Belyaev, A. P. Matafonov, V. I. Vinogradov, V. P. Krainov, V. S. Lisitsa, A. S. Roussetski, G. N. Ignatyev, and V. P. Adrianov, Phys. Rev. E 72, 026406 (2005).

[3] S. Kimura, A. Anzalone, and A. Bonasera, Phys. Rev. E 79, 038401 (2009).

[4] C. Labaune, C. Baccou, S. Depierreux, C. Goyon, G. Loisel, V. Yahia, and J. Rafelski, Nat. Commun. 4, 2506 (2013).

[5] A. Picciotto, D. Margarone, A. Velyhan, P. Bellutti, J. Krasa, A. Szydlowsky, G. Bertuccio, Y. Shi, A. Mangione, J. Prokupek, A. Malinowska, E. Krousky, J. Ullschmied, L. Laska, M. Kucharik, and G. Korn, Phys. Rev. X 4, 031030 (2014).

[6] L. Giuffrida, F. Belloni, D. Margarone, G. Petringa, G. Milluzzo, V. Scuderi, A. Velyhan, M. Rosinski, A. Picciotto, M. Kucharik, J. Dostal, R. Dudzak, J. Krasa, V. Istokskaia, R. Catalano, S. Tudisco, C. Verona, K. Jungwirth, P. Bellutti, G. Korn, and G. A. P. Cirrone, Phys. Rev. E 101, 013204 (2020)

[7] D. Margarone, A. Morace, J. Bonvalet, Y. Abe, V. Kantarelou, D. Raffestin, L. Giuffrida, P. Nicolaï, M. Tosca, A. Picciotto, G. Petringa, G. A. P. Cirrone, Y. Fukuda, Y. Kuramitsu, H. Habara, Y. Arikawa, S. Fujioka, E. D’Humieres, G. Korn, and D. Batani, Front. Phys. 8, 343 (2020).

[8] W. M. Nevins and R. Swain, Nucl. Fusion 40, 865 (2000)

[9] C. Labaune, C. Baccou, V. Yahia, C. Neuville, and J. Rafelski, Sci. Rep. 6, 21202 (2016).

[10] J. Jung and T. Suk, Appl. Phys. Lett. 105, 223507 (2014).

[11] L. Giuffrida, D. Margarone, G. A. P. Cirrone, A. Picciotto, G. Cuttone, and G. Korn, AIP Adv. 6, 105204 (2016).

[12] G. A. P. Cirrone, L. Manti, D. Margarone, G. Petringa, L. Giuffrida, A. Minopoli, A. Picciotto, G. Russo, F. Cammarata, P. Pisciotta, F. M. Perozziello, F. Romano, V. Marchese, G. Milluzzo, V. Scuderi, G. Cuttone, and G. Korn, Sci. Rep. 8, 1141 (2018).

[13] K. Hideghety, S. Brunner, A. Cheesman, E. Szabo, R. Polanek, D. Margarone, T. Tokes, and K. Mogyorosi, Anticancer Res. 39, 2265 (2019).

[14] R. F. Barth, A. H. Soloway, and R. G. Fairchild, Cancer Res. 50, 1061 (1990).
[15] J. Jung, D-K. Yoon, B. Barraclough, H. Chang Lee, T. Suk Suh, and B. Lu, Oncotarget 8, 39774 (2017).

[16] S. Miyatake, S. Kawabata, K. Yokoyama, T. Kuroiwa, H. Michiue, Y. Sakurai, H. Kumada, M. Suzuki, A. Maruhashi, M. Kirihata, and K. Ono, J. Neurooncol. 91, 199 (2009).

[17] S. Kurosawa, H. Kubo, K. Ueno, S. Kabuki, M. Takahashi, K. Taniue, N. Higashi, K. Miuchi, T. Tanimor, D. Kim, and J. Kil, Curr. Appl. Phys. 12, 364 (2012).

[18] G. Petringa, G. A. P. Cirrone, C. Caliri, G. Cuttone, L. Giuffrida, G. La Rosa, R. Manna, L. Manti, V. Marchese, C. Marchetta, D. Margarone, G. Milluzzo, A. Picciotto, F. Romano, F. P. Romano, A. D. Russo, G. Russo, D. Santonocito, and V. Scuderi, J. Instrum. 12, C03059 (2017).

[19] D. Margarone, A. Picciotto, A. Velyhan, J. Krasa, M. Kucharik, A. Mangione, A. Szydlowsky, A. Malinowska, G. Bertuccio, Y. Shi, M. Crivellari, J. Ullschmied, P. Bellutti, and G. Korn, Plasma Phys. Controlled Fusion 57, 014030 (2015).

[20] K. Szkliniarz, M. Sitarz, R. Walczak, J. Jastrzebski, A. Bilewicz, J. Choinski, A. Bakubowski, A. Majkowska, A. Stolarz, A. Trzcinska, and W. Zipper, Appl. Radiat. Isot. 118, 182 (2016).

[21] F. Haddad, L. Ferrer, A. Guertin, T. Carlier, N. Michel, J. Barbet, and J.-F. Chatal, Eur. J. Nucl. Med. Mol. Imaging 35, 1377 (2008).

[22] A. Morace, N. Iwata, Y. Sentoku, K. Mima, Y. Arikawa, A. Yogo, S. Tosaki, X. Vaisseau, Y. Abe, S. Kojima, S. Sakata, M. Hata, S. Lee, K. Matsuo, N. Kamitsukasa, T. Norimatsu, J. Kawanaka, S. Tokita, N. Miyanaga, H. Shiraga et al. Nat. Commun. 10, 2995 (2019).

[23] A. Macchi, M. Borghesi, and M. Passoni, Rev. Mod. Phys. 85, 751 (2013).

[24] N. Rabhi, D. Batani, G. Boutoux, J.-E. Ducret, K. Jakubowska, I. Lantuejoul-Thfoin, C. Nauraye, A. Patriarca, A. Sa'd, A. Semsoum, L. Serani, B. Thomas, and B. Vauzour, Rev. Sci. Instrum. 88, 113301 (2017).

[25] T. Bonnet, M. Comet, D. Denis-Petit, F. Gobet, F. Hannachi, M. Tarisien, M. Versteegen, and M. M. Aléonard, Rev. Sci. Instrum. 84, 103510 (2013).

[26] www.ct.infn.it/en/. 
[27] J. Derouillat, A. Beck, F. Pérez, T. Vinci, M. Chiaramello, A. Grassi, M. Fle, G. Bouchard, I. Plotnikov, N. Aunai, J. Dargent, C. Riconda, and M. Grech, Comput. Phys. Commun. 222, 351 (2018).

[28] F. Sommerer, K. Parodi, A. Ferrari, K. Poljanc, W. Enghardt, and H. Aiginger, Phys. Med. Biol. 51, 4385 (2006).

[29] D. Batani R. Jafer, M. Veltcheva, R. Dezulian, O. Lundh, F. Lindau, A. Persson, K. Osvay, C.-G. Wahlström, D. C. Carroll, P. McKenna, A. Flacco, and V. Malka, New J. Phys. 12, 045018 (2010).
[30] Malka, J. Faure, S. Fritzler, and M. Manclossi, Rev. Sci. Instrum. 77, 03B302 (2006).

[31] F. Geser and M. Valente, Appl. Radiat. Isot. 151, 96 (2019).

[32] F. Fiuza, A. Stockem, E. Boella, R. A. Fonseca, L. O. Silva, D. Haberberger, S. Tochitsky, C. Gong, W. B. Mori, and C. Joshi, Phys. Rev. Lett. 109, 215001 (2012).

[33] https://www-nds.iaea.org/exfor/.

[34] H. Becker, C. Rolfs, and H. Trautvetter, Z. Phys. A: At. Nucl. 327, 341 (1987).

[35] A. Macchi, F. Cattani, T. V. Liseykina, and F. Cornolti, Phys. Rev. Lett. 94, 165003 (2005). 